ter) attempted to explain the evolution of the Venus atmosphere, comparing the process with the evolution of the Earth's atmosphere. The two seem greatly different today, chiefly because of the lack of water on Venus. Meadows pointed out that the difference could be explained in terms of a photodissociation process operating strongly on Venus to break water down (through interactions involving carbon dioxide) to oxygen and hydrogen, the latter escaping into space. But he said further that there is no need to invoke this process, and that he would be prepared to accept that Venus has had a predominately carbon dioxide atmosphere "from the start".

In this model, $\mathrm{CO}_{2}$ is produced by the effect of heat on carbonates and silicates in the Venus rocks. Several reversible reactions involving these compounds include carbon dioxide as a product, and the amount of $\mathrm{CO}_{2}$ present would then depend just on the temperature. Once a little atmosphere existed, the greenhouse effect would allow conditions very like those on Venus today to be established, given the boundary condition of the present output of the Sun. Since the Sun is slowly warming up, this suggests an increase in the density of the Venus atmosphere in future. The initial wisp of atmosphere needed to start the process could either come from a burst of volcanism, or slower outgassing.

Venus without its atmosphere must have been much the same as Mercury is today, and J. E. Guest (University College, London) described with the aid of Mariner 10 pictures the surface of Mercury and impact theories of the cratering. Much of this material formed something of a travelogue; for the mixed audience, the most interesting feature of the Mariner 10 pictures is that signs of compressional faulting indicate that the planet has shrunk by one or two kilometres since its formation.

The new studies of the inner planets support Runcorn's attempts to describe Mercury, Venus, Earth, Moon and Mars in one relatively simple model. If Mars and Mercury are both cratered like our Moon, then surely Earth and Venus have been through the same bombardment, providing a clue to events early in the development of the Solar System. If tectonic processes have occurred on Mars (and shrinkage on Mercury) geophysicists may gain a better insight into continental drift on Earth. And if satisfactory models of the Venus and Mars atmospheres are developed, this must help in understanding the workings of our own atmosphere.

As long ago as 1958, the RAS and
RMetSoc held a joint meeting on planetary atmospheres. The way things are going, it certainly will not be a further 17 years before another tripartite meeting about the inner planets is held.

\section{The sea as a chemical system}

from Peter S. Liss

The Dahlem Workshop on the Nature of Seawater was held in Berlin on March 10-15, under the chairmanship of Professor E. D. Goldberg (Scripps Institution of Oceanography).

IN comparison with the solutions normally studied by pure chemists, seawater is a very complicated medium. Not only is its ionic strength high ( $\sim 0.7 \mathrm{M})$, but the ions in solution represent virtually all the naturally occurring elements, with concentrations of different elements ranging over at least twelve orders of magnitude. Funthermore, many of the dissolved species are involved in biological processes and reactions at the air-sea and water-sediment interfaces. Pure and marine chemists met at the workshop to discuss techniques and concepts in chemistry which could deepen our understanding of seawater and of chemical reactions occurring in the marine enviromment.

The ionic strength and composition of seawater are very different from those of the aqueous media normally used for the determination of stability constants. Calculation of the effect of seawater yields only approximate values and for more accurate work, the discussion group which dealt with this topic concluded that it is much better to employ the ionic medium scale, that is, to use seawater (real or artificial) as the reference solution. A self-consistent set of conventions was proposed and its use will allow the development of uniform and thermodynamically rigorous data for seawater equilibria. The new approach has many important implications for marine chemistry, including the redefinition of $p H$ and redox potential, and the use of reference solutions which closely match seawater in ionic composition for $p \mathrm{H}$ and ionselective electrode measurements. It will obviate the need to use single ion activity coefficients and lead to phasing out of the scheme involving 'apparent' stability constants, which has served chemical oceanographers well for many years.

Sample collection is a perennial problem in seawater chemistry and is especially acute for the sea surface microlayer. Most techniques available at present involve dipping a screen or plate into the water to collect a layer approximately $200 \mu \mathrm{m}$ thick. For the rapid collection of milligram quantities of material, consideration should be given to the technique of foam fractionation in which air or nitrogen is bubbled through the water to create a foam which, because of its large surface-tovolume ratio, will very efficiently scavenge surface-active molecules.

Less than $10 \%$ of the dissolved organic matter in seawater has been characterised and an important objective should be the identification of the remainder in representative samples from the deep ocean, above the thermocline and surface microlayer. Techniques are probably available for detailed identification, but these tend to be complex and expensive. Characterisation into broad classes of compounds and major functional groups is simpler and will yield much important information.

Our present knowledge of the particulate fraction in marine waters is rudimentary. For instance, in order to measure size distribution of particles the change in solution conductance due to the presence of the particles is often employed. However, using this method only part of the size spectrum can be examined at any one time and what is really measured is not the particles themselves but a complicated function of the relative conductivities of the particle and the solution. A much more promising technique is to use singleparticle scattering counters. These devices, which use laser sources, have already been employed in studies of hydrosols and aerosols.

Though much is known about 'pure' solids (for example the role of electrical double layers, ion exchange reactions, particle-particle interactions), in the marine environment such properties are certainly very substantially altered because the particles have an organic coating. At present very little is known about the properties and composition of such layers, although it should be possible to use techniques such as microelectrophoresis and potentiometric titration to identify some of the major organic functional groups in the coatings, as well as in organic particles more generally.

The goal of the workshop was certainly achieved in a number of areas of seawater chemistry; reported here are only a few of the topics discussed. Where less progress was made it was generally due to the inability of the marine chemists to state the problems in a meaningful way, rather than any reluctance on the part of the chemists to get to grips with the complexity of the sea as a chemical system. 